\title{
Osteonecrose mandibular relacionada com bifosfonatos orais em paciente idosa polimedicada
}

\author{
Biphosphonate-related osteonecrosis of the jaw in an old patient with oral bisphosphonate exposure
}

\section{Osteonecrosis mandibular relacionada con la toma de bifosfonatos orales en paciente anciana polimedicada}

Leticia Simao Aiex. Servicio Andaluz de Salud, Distrito Sanitario de Jaén. Jaén, Espanha. leticia.aiex@gmail.com (Autora correspondente)

Maria Villa Juárez Jimenez. Servicio Andaluz de Salud, Distrito Sanitario de Jaén. Jaén, Espanha. prettywoman_mvjj@hotmail.com Alejandro Pérez Milena. Servicio Andaluz de Salud, Centro de Salud El Valle. Jaén, Espanha. alpemi@gmail.com

\section{Resumo}

A polifarmácia define-se como o consumo de cinco ou mais fármacos simultâneos durante um período de tempo determinado e é cada vez mais frequente em pessoas maiores de 65 anos. A osteoporose é a alteração metabólica óssea mais frequente nos países ocidentais e o segundo problema sanitário no mundo, depois das doenças cardiovasculares. Sua maior complicação é a fratura por fragilidade, afetando mais mulheres que homens. A prevenção de fraturas por osteoporose é feita com diversos tipos de remédios e os mais usados são os bifosfonatos. Estes são fármacos com amplo uso em Atenção Primária, principalmente para osteoporose pós-menopáusica. Apesar de serem bem tolerados, não estão isentos de efeitos secundários, sendo um dos mais graves a osteonecrose mandibular, como ocorre no caso exposto neste trabalho. Por este e outros motivos, sempre é importante ponderar risco-benefício de forma individualizada antes de começar qualquer tratamento e, principalmente, quando se trata a de pacientes polimedicados.

\section{Abstract}

Polypharmacy is defined as consumption of five or more concomitant drugs over a period of time, and its incidence is more common in people aged over 65 years. Osteoporosis is the most frequent metabolic bone disease in Western countries and the second most frequent health problem worldwide after cardiovascular diseases. Its main complication is fracture caused by bone fragility, and it affects more women than men. Various treatments are available to prevent them, and one of the most commonly used treatments is bisphosphonate therapy. These drugs are widely used as first-line therapy, especially in postmenopausal osteoporosis. Although biphosphonates are generally well tolerated, they are not free of side effects, and one of the most serious ones is osteonecrosis of the jaw, as shown in the following clinical case. For these and other reasons, it is always important to perform risk-benefit analysis individually before starting any treatment, especially in patients with polypharmacy.
Palavras-chave:

Osteoporose Pós-Menopausa Bifosfonatos

Osteonecrose

Mandíbula

Polimedicação

\section{Keywords:}

Osteoporosis, Postmenopausal Bisphosphonates

Osteonecrosis

Mandible

Polypharmacy
Como citar: Aiex LS, Jimenez MVJ, Milena AP. Osteonecrose mandibular relacionada com bifosfonatos orais em paciente idosa polimedicada. Rev Bras Med Fam Comunidade. 2015;10(36):1-7. http://dx.doi.org/10.5712/rbmfc10(36)748
Fonte de financiamento: declaram não haver. Parecer CEP: não se aplica. Conflito de interesses: declaram não haver. Procedência e revisão por pares: revisado por pares. Recebido em: 30/04/2013. Aprovado em: 27/04/2015. 


\section{Resumen}

Polimedicación es el consumo de cinco o más fármacos de forma concomitante durante un período de tiempo determinado y aparece más frecuentemente en personas mayores de 65 años. La osteoporosis es la enfermedad metabólica ósea más frecuente en los países occidentales y el segundo problema sanitario en el mundo después de las enfermedades cardiovasculares. Su mayor complicación es la fractura por fragilidad, afectando más a mujeres que hombres. Para prevenirlas se emplean diversos tipos de medicinas, y las más utilizadas son los bifosfonatos. Estos son medicamentos ampliamente empleados en la Atención Primaria, principalmente en osteoporosis posmenopáusica. A pesar de ser generalmente bien tolerados, no están libres de efectos adversos, siendo uno de los más graves la osteonecrosis mandibular, como ocurre en el caso clínico expuesto. Por estos y otros motivos, siempre es importante valorar los riesgos-beneficios de forma individualizada antes de empezar cualquier tratamiento, y más cuando se trata de pacientes polimedicados.

\section{Palabras clave:}

Osteoporosis Postmenopáusica Bifosfonatos

Osteonecrosis

Mandíbula

Polifarmacia

\section{Introdução}

A polifarmácia é definida pelo Serviço Nacional de Saúde da Espanha como o consumo de cinco ou mais fármacos de maneira concomitante durante um período superior a três semanas. ${ }^{1}$

Essa situação é vista com frequência cada vez maior: estima-se que aproximadamente metade dos pacientes que frequentam um centro de Atenção Primária encaixa-se nessa definição. ${ }^{2}$ É mais frequente nos pacientes idosos. Está associada a um maior risco de efeitos colaterais e interações medicamentosas devido às mudanças na farmacodinâmica e farmacocinética dos medicamentos com o passar da idade..$^{3,4}$

Outro problema relevante são as fraturas por osteoporose, que ocorrem principalmente em pessoas com mais de 70 anos. ${ }^{5,6}$

Segundo a Organização Mundial da Saúde $(\mathrm{OMS})^{7}$ e a Conferência do National Institute of Health, ${ }^{8}$ a osteoporose define-se como doença esquelética caracterizada por uma resistência óssea diminuída que predispõe ao aumento do risco de fratura, afetando a integridade da densidade e a qualidade do osso. ${ }^{7,8}$

É considerada a alteração metabólica mais frequente nos países ocidentais e o segundo problema sanitário assistencial no mundo, depois das doenças cardiovasculares. ${ }^{8} \mathrm{Na}$ Espanha, afeta a mais de três milhões de pessoas, aumentando a incidência com a idade. ${ }^{9} \mathrm{~A}$ complicação mais temível é a fratura osteoporótica ou por fragilidade que afeta a mais de $70 \%$ das mulheres a partir dos 80 anos. ${ }^{7-9}$ No estudo conduzido no Brasil, BRAZOS (The Brazilian Osteoporosis Study), encontrou-se prevalência de 6\% de osteoporose e $15 \%$ de fraturas por osteoporose. ${ }^{10}$

Atualmente, existem diversas alternativas de tratamento para prevenir as fraturas por osteoporose. Nos últimos anos, os bifosfonatos por via oral, com suas formas de administração semanal e mensal, constituíram a família de remédios mais usados. ${ }^{11}$ No entanto, seu uso pode gerar um alto custo econômico, além de estar relacionado com diversos efeitos colaterais, como erosões e úlceras crônicas da mucosa oral, úlcera gástrica, esofagite, estenose esofágica, fratura atípica (subtrocantéreas e diafisárias), dor musculoesquelética e fibrilação atrial. Além destas, também encontrou-se outra complicação grave: a osteonecrose mandibular (ONM), ${ }^{12}$ que pode ser mais dificilmente diagnosticada em pacientes idosos polimedicados, como ocorre no caso clínico exposto.

A incidência desta patologia, que era baixa, com o considerável aumento da utilização dos bifosfonatos na prevenção de fratura por osteoporose - principalmente em pacientes polimedicados -, aumentou de forma considerável. 


\section{Descrição do caso}

Mulher espanhola, residente na província de Jaén (Andaluzia, Espanha), de 83 anos de idade que, em setembro de 2009, foi atendida por ortopedista devido a quadro de poliartralgia mecânica, sem melhora com analgésicos de primeira e segunda linha. Foi diagnosticada, através de radiografia dorso-lombar de escoliose, espondiloartrose e osteopenia severa lombar e moderada na bacia, indicando-se que começasse tratamento com paracetamol, fentanila transdérmica, ácido ibandrônico 150mcg retard mensal, além de carbonato de cálcio e colecalciferol diários.

Também vinha sendo acompanhada por seu médico de família para controle de doenças que constituem fatores de risco cardiovascular (hipertensão arterial, diabetes mellitus (DM) tipo 2, dislipidemia, obesidade, insuficiência renal crônica leve), as quais eram tratadas com doses diárias de enalapril, hidroclorotiazida, glicazida, metformina e sinvastatina. Além disso, devido à presença de úlcera duodenal e glaucoma, usava omeprazol e timolol tópico, sendo também portadora de prótese dentária.

Desde julho de 2010, consultou-se com seu médico, em diversas ocasiões, por dor na gengiva acompanhada de abscesso maxilar inferior direito. Em primeira instância, essas reclamações foram atribuídas ao uso de dentadura e infecção dentária. Ela foi vista tanto pelo médico de família quanto pelo dentista, que indicaram tratamentos como antibióticos orais (amoxicilina e amoxicilina-clavulanato), anti-inflamatórios e diversos enxágues com antissépticos tópicos, sem êxito. Até que, em junho de 2011, realizou-se nova inspeção da cavidade oral, que evidenciou exposição óssea do corpo mandibular direito, acompanhada de lesão ulcerada no extremo interno do mesmo, e inflamação local intensa. Nesse momento, fez-se necessário o diagnóstico diferencial entre cárie, gengivite, periodontite, prótese dental mal colocada e lesão óssea do maxilar. Solicitou-se uma ortopantomografia, que mostrou imagem hipodensa no terço inferior do corpo mandibular direito de aproximadamente 1 centímetro de diâmetro (Figura 1a). Diante disso, o tratamento com bifosfonatos foi suspenso imediatamente pelo médico de família, e a paciente foi encaminhada para o cirurgião maxilofacial.

Em julho de 2011, foi avaliada pela Cirurgia Maxilofacial, onde foi solicitada uma tomografia computadorizada (TC) de mandíbula (Figura 2). Os resultados mostraram uma lesão de 1,3×1,2 centímetros no terço médio do corpo mandibular direito, com lesões esclerosadas no interior e destruição da cortical interna, além de reação esclerótica no resto do corpo mandibular, sendo diagnosticada de lesão inespecífica de tempo de evolução incerta e duvidosa origem neoplásica. Com esses resultados, e depois de comprovar as características e extensão da lesão, realizou-se diagnóstico final de osteonecrose mandibular provocada por bifosfonatos orais (ácido ibandrônico). Assim, realizou-se a extirpação do osso necrótico, com biópsia da lesão lítica. O diagnóstico anatomopatológico foi de actinomicose.

Atualmente, depois de duas revisões pós-cirúrgicas (janeiro/2012 e janeiro/2013), a paciente encontra-se assintomática, existindo uma pequena regeneração óssea (Figura 1b) com lesão totalmente coberta pela mucosa. Os sintomas desapareceram e mantém-se o tratamento para osteoporose mediante ingestão suplementar de cálcio e atividade física adequada para a idade. 


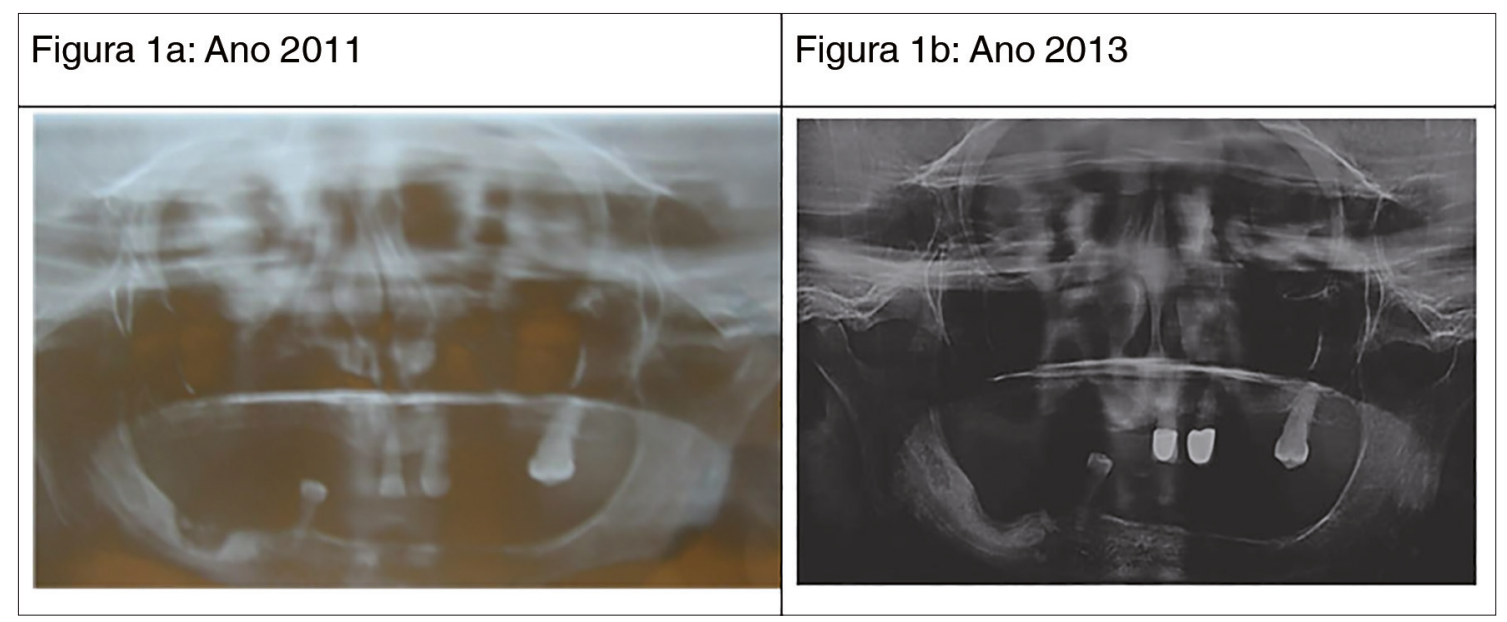

Figura 1. Evolução radiológica.

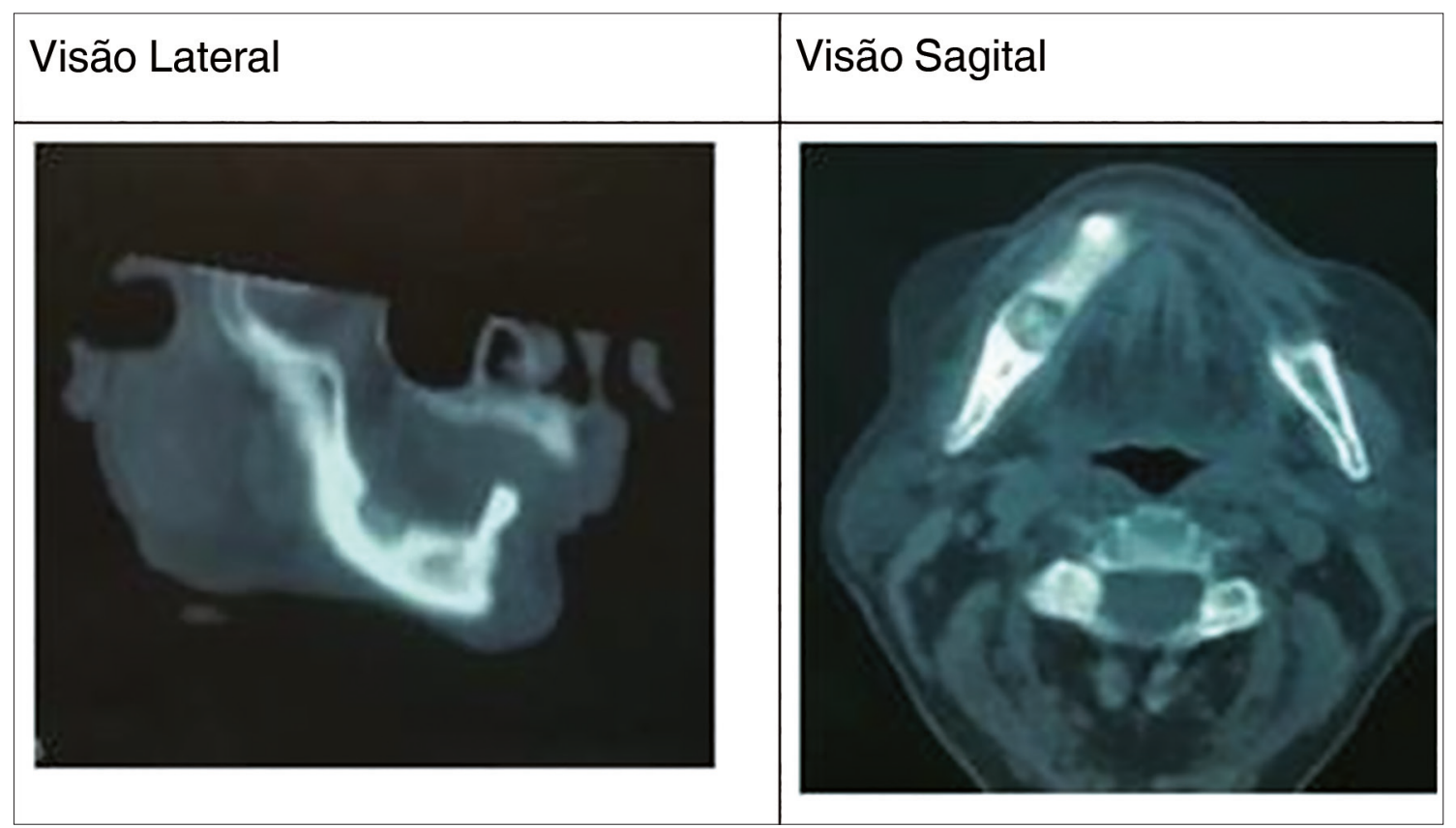

Figura 2. Tomografia computadorizada (TC) cervical, realizada em julho de 2011.

\section{Discussão}

As fraturas por fragilidade, em mulheres com osteoporose, representam um problema de saúde pública mundial, com inumeráveis repercussões sociosanitárias e económicas. Por isto, antes de instaurar qualquer tipo de tratamento, é importante realizar um correto diagnóstico. ${ }^{13}$

Em 1994, a OMS definiu alguns critérios diagnósticos de osteoporose baseados nos valores da densitometria óssea (DMO) - osteoporose se DMO $\leq 2,5 \mathrm{DE}$. Considerando a DMO, encontraram-se vários fatores de risco de fratura por fragilidade exclusivos das mulheres, como a menopausa precoce e o status hipoestrogênico, tal como anorexia nervosa, excesso de exercício, câncer de mama. ${ }^{14}$

Além desses, existem muitos outros fatores de risco para a perda óssea e, consequentemente, fraturas por fragilidade, como a idade avançada (o mais importante), baixo índice de massa corporal $(<19)$, história prévia e/ou familiar de fraturas, ser fumante, elevado consumo de álcool. ${ }^{15}$ 
Atualmente, indica-se a realização de DMO em mulheres entre 50 e 64 anos que possuam alguns destes fatores de risco ou que apresentem: fratura por fragilidade depois dos 40 anos, uso prologando de corticoides (>3 meses), baixo peso corporal ( $<60 \mathrm{~kg}$ ) ou emagrecer $>10 \%$ do peso corporal aos 25 anos, artrite reumatoide. ${ }^{16}$ No entanto, apesar de existir osteoporose densitométrica, estima-se que menos de um terço do risco de fratura deve-se ao valor densitométrico, sendo a idade, por si só, sete vezes mais importante. ${ }^{16}$

Para calcular o risco de fratura, existem muitas escalas válidas, sendo a mais destacada a FRAX, que permite calcular o risco de fratura aos 10 anos, porém, esta não dá recomendações sobre quando iniciar o tratamento farmacológico. Existem outros modelos, como o de Rotterdam, que dão algumas recomendações mais específicas sobre o momento de início do tratamento. ${ }^{15}$ Porém, nunca se deve esquecer que apesar das escalas serem úteis e proporcionarem informações de orientação clínica geral, o tratamento deve ser visto de forma individual em cada caso.

O tratamento preventivo é composto por dieta, exercício físico, cessação do tabagismo, prevenção de quedas, cautela na prescrição medicamentosa, além de medidas farmacológicas. ${ }^{17}$

É sabido que o tratamento farmacológico da osteoporose é custo efetivo, e deve-se instaurar em todos os casos de prevenção secundária, ou seja, naquelas mulheres pós-menopáusicas com osteoporose que tiveram uma fratura por fragilidade, e também nos casos de prevenção primária sempre que exista um risco alto (>20\% de probabilidade de fratura nos próximos 10 anos) ou risco médio (10-20\% de probabilidade) com osteoporose densitometrica. ${ }^{15}$

Porém, estas recomendações têm uma limitação, pois apesar das mulheres com mais de 80 anos constituírem um grupo de grande risco, existem poucos dados da eficiência dos tratamentos farmacológicos neste grupo, pois, em geral, estas não são incluídas nos estudos. ${ }^{15}$ Portanto, a paciente descrita anteriormente não foi corretamente diagnosticada de osteoporose, pois a radiografia não é um método de utilidade para tal. Além disso, não se calculou o risco de fratura, nem outros riscos de iniciar novo tratamento a uma paciente idosa polimedicada. Assim, pode-se afirmar que não existiam dados suficientes para iniciar tratamento farmacológico.

Apesar de o Serviço Andaluz de Saúde recomendar o uso de alendronato como tratamento de primeira linha para a osteoporose, 1/5 das mulheres pós-menopáusicas com osteoporose na Espanha usam ibandronato mensal como bifosfonato eletivo. ${ }^{17}$ Sua vantagem encontra-se na comodidade de administração, melhorando a aderência ao tratamento, principalmente em pacientes polimedicados. Em estudos de prevenção secundária, observou-se que reduz as fraturas vertebrais em $62 \%$, com um NNT=20. No entanto, não está demostrado que realmente seja mais eficaz. Além disto, existem poucos estudos cujo objetivo seja avaliar sua eficácia e segurança na população de idosos, e em prevenção primária de fraturas por fragilidade. ${ }^{17,18}$

Demonstrou-se que o alendronato é capaz de aumentar a densidade mineral óssea e diminuir em quase metade os casos de fratura vertebral (prevenção primaria) nas mulheres com mais de 65 anos, enquanto seu efeito nas demais fraturas é mais controverso. ${ }^{8,18}$ Em estudos de prevenção secundária, o alendronato demonstrou reduzir as fraturas vertebrais radiológicas (NNT=15), fraturas vertebrais sintomáticas (NNT=37) e outras fraturas (NNT=36).

ONM secundária aos bifosofonatos é uma osteomielite crônica, lenta, de diagnóstico recente. Observa-se a presença de lesão ulcerada na mucosa oral com exposição óssea de mais de 8 semanas de evolução e curso insidioso. ${ }^{19} \mathrm{O}$ diagnóstico é feito pela presença dessa lesão ulcerada, uso de bifosfonatos 
e ausência de radiação no território maxilofacial, e existem três estágios: 1) assintomático; 2) dor e provas clínicas de infecção; 3) complicações: ulceração da mucosa e exposição do osso desvitalizado, infecção de tecidos moles, perda de dentes e parestesias faciais..$^{20,21}$ A etiopatogenia parece basear-se na presença de tecido hipóxico, hipocelular e hipovascular secundário ao potencial anti-angiogênico dos bifosfonatos, assim como à inibição da troca óssea que provocam, o que faz com que o osso seja mais suscetível a infecções que acabem em necrose. ${ }^{20}$

A incidência de ONM por bifosfonatos orais está estimada entre $0,01-0,04 \% .{ }^{22} \mathrm{O}$ risco desta complicação parece aumentar quando a duração do tratamento supera os três anos e a maioria dos casos descritos tinha uma extração dentária prévia com cicatrização por segunda intenção, ${ }^{21}$ fato que não ocorreu no caso descrito anteriormente. No entanto, dado o elevado número de pacientes tratados com bifosfonatos orais para prevenção de fraturas por osteoporose, é esperado que esse efeito adverso seja cada vez mais frequente, visto que os bifosfonatos apresentam uma longa meia vida nos ossos (11 anos)..$^{21,22}$

Assim, o tratamento da osteoporose deve ser individualizado, indicando os bifosfonatos de acordo com o risco de fraturas e a necessidade de prevenção farmacológica, com uma adequada avaliação dos riscos/benefícios. Não é aconselhável manter esse tratamento por mais de cinco anos, devido ao aumento no risco de efeitos adversos graves, sem aumentar suas características protetoras sobre fraturas. . 20-22 $^{2}$ Alguns autores recomendam retirar o bisfosfonato três meses antes de qualquer cirurgia oral, sempre que seja clinicamente possível. ${ }^{8,17,19,21}$

Essas recomendações devem ser especialmente lembradas pelos médicos de família para evitar complicações. Deve-se aconselhar uma correta higiene bucal e o abandono de tabagismo e consumo de álcool. Também deve-se lembrar do conceito de prevenção quaternária, cujo objetivo é evitar o sobrediagnóstico e sobretratamento, ou seja, diminuir a iatrogenia. Deve-se evitar a promoção de doenças e prevenir a medicalização da sociedade. Para isso, não se podem confundir fatores de risco com doenças, para não provocar problemas no presente ao prevenir eventos futuros.

Como conclusão, é importante individualizar o risco-beneficio para justificar começar um tratamento farmacológico, principalmente quando estamos diante de pacientes idosos ou polimedicados. ${ }^{9}$ Sempre que haja suspeita da doença, devem-se retirar os bifosfonatos, além de aumentar as medidas de vigilância destes pacientes.

Por último, deve-se lembrar que além do uso de remédios não ser inócuo, seus efeitos adversos são mais frequentes em pacientes idosos polimedicados, já que estes apresentam uma resposta aos medicamentos diferente às pessoas de menor idade, e devido às possíveis interações farmacológicas. No entanto, a idade não deve ser uma barreira para começar um tratamento sempre que esteja realmente indicado.

\section{Referências}

1. Villafaina Barroso A, Gavilán Moral E. Pacientes polimedicados frágiles, un reto para el sistema sanitário. Inf Ter Sist Nac Salud. 2011;35(4):114-23.

2. Molina López T, Caraballo Camacho Mde L, Palma Morgado D, López Rubio S, Domínguez Camacho JC, Morales Serna JC. Prevalencia de polimedicación y riesgo vascular en la población mayor de 65 años. Aten Prim. 2012;44(4):216-22. DOI: http://dx.doi.org/10.1016/j.aprim.2011.03.010

3. Carreño José V, Gayo Loredo ML, Pérez Manzano F, Gómez Ortega Al, Acosta Ferrer M. Interacciones farmacológicas en población polimedicada. Aten Primaria. 2008;40(11):582-3. DOI: http://dx.doi.org/10.1157/13128576 
4. Steinman MA, Landefeld CS, Rosenthal GE, Berthenthal D, Sen S, Kaboli PJ. Polypharmacy and prescribing quality in older people. J Am Geriatr Soc. 2006;54(10):1516-23 DOI: http://dx.doi.org/10.1111/j.1532-5415.2006.00889.x

5. Brozoski MA, Traina AA, Deboni MC, Marques MM, Naclério-Homem Mda G. Bisphosphonate-related osteonecrosis of the jaw. Rev Bras Reumatol. 2012;52(2):265-70. DOI: http://dx.doi.org/10.1590/S0482-50042012000200010

6. González López-Valcárcel B, Sosa Henríquez M. Estimación del riesgo de fractura osteoporótica a los 10 años para la población española. Med Clin (Barc). 2013;140(3):104-9. DOI: http://dx.doi.org/10.1016/j.medcli.2011.11.030

7. Osteoporosis fragility fracture risk: Guideline consultation. NICE Guidance; 2012. Disponível em: http://guidance.nice.org.uk

8. Moro-Alvarez MJ, Díaz-Curiel M. Diagnóstico y tratamiento de la osteoporosis en mayores de 75 años. Rev Esp Geriatr Gerontol. 2010;45(3):141-9. DOI: http://dx.doi.org/10.1016/j.regg.2009.11.003

9. Guerra-García MM, Rodríguez-Fernández JB, Puga-Sarmiento E, Charle-Crespo MÁ, Gomes-Carvalho CS, PrejigueiroSantás A. Incidencia de la fractura de cadera osteoporótica en Galicia en relación con la dispensación de medicamentos con indicación en su prevención y tratamiento. Aten Primaria. 2011;43(2):82-8. DOI: http://dx.doi.org/10.1016/j. aprim.2010.04.010

10. Brandão CMR, Machado GPM, Acurcio FA. Análise farmacoeconômica das estratégias de tratamento da osteoporose em mulheres na pós-menopausa: uma revisão sistemática. Rev Bras Reumatol. 2012;52(6):912-37. DOI: http://dx.doi. org/10.1590/S0482-50042012000600010

11. Wells G, Cranney A, Peterson J, Boucher M, Shea B, Welch V, et al. Alendronato para la prevención primaria y secundaria de las fracturas osteoporáticas en mujeres posmenopáusicas (revisión Cochrane traducida) [Internet]. Cochrane Database Syst Rev. 2008 Jan 23;(1):CD001155 [Acesso 14 Ago 2015]. Disponível em: http://www.cochrane.org/es/CD001155/ alendronato-para-la-prevencion-primaria-y-secundaria-de-las-fracturas-osteoporoticas-en-mujeres-posmenopausicas.

12. Filleul O, Crompot E, Saussez S. Bisphosphonate-induced osteonecrosis of the jaw: a review of 2,400 patient cases. J Cancer Res Clin Oncol. 2010;136(8):1117-24. DOI: http://dx.doi.org/10.1007/s00432-010-0907-7

13. González-Macías J, Guañabens G, Gómez Alonso C, Del Río Barquero L, Muñoz Torres M, Delgado M, et al. Guía de práctiva clínica en la osteoporosis postmenopáusica, glucocorticoidea y del varón. Sociedad Española de Investigación Ósea y del metabolismo Mineral. Rev Clin Esp. 2008;208(Supl 1):1-24.

14. Areas 1, 2, 3, 5 y 7 de Atencion Primaria. Servicio Madrileno de Salud. Prevencion y tratamiento de la osteoporosis posmenopausica: prevencion de fracturas. Notas Farmacoterapeuticas. 2005;12(5):9-16.

15. Dirección General de Farmacia y Productos Sanitarios. Recomendaciones para la valoración y tratamiento de la osteoporosis primaria en mujeres de la Comunidad de Madrid. Consejería de Sanidad; 2007.

16. Papaioannou A, Morin S, Cheung AM, Atkinson S, Brown JP, Feldman S, et al. ; Scientific Advisory Council of Osteoporosis Canada. 2010 clinical practice guidelines for the diagnosis and management of osteoporosis in Canada: summary. CMAJ. 2010;182(17):1864-73. DOI: http://dx.doi.org/10.1503/cmaj.100771

17. Vicente Molinero A, Lou Arnal S, Medina Orgaz E, Muñoz Jacobo S, Antonio Ibáñez Estrella J. Osteoporosis treatment with biphosphonates: Approaches to care reality. Aten Primaria. 2011;43(2):95-9. PMID: 20541844 DOI: http://dx.doi. org/10.1016/j.aprim.2010.04.008

18. Fernandez Avila DG, Mora C, Reyes Sanmiguel E, Londoño JD, Santos Moreno P, Valle-Oñate R. Tratamiento farmacologico de la osteoporosis postmenopáusica. Rev Colomb. Reumatol. 2010;17(2):96-110

19. Arroyo Pineda V, Tofino Gonzalez I, de la Hija Díaz MB. Ibandronato Mensal para la Osteoporosis. Hoja de Evaluación de medicamentos de Castilla La Mancha. Sescam. 2008;9(7).

20. Arranz Caso JA, Flores Ballester E, Ngo Pombe S, López Pizarro V, Dominguez-Mompello JL, Restoy Lozano A. Bisphosphonate related osteonecrosis of the jaw and infection with Actinomyces. Med Clin (Barc). 2012;139(15):676-80. DOI: http://dx.doi.org/10.1016/j.medcli.2012.05.039

21. Junquera LM. Comisión científica de la SECOM: Acero J, Burgueño M, de Vicente JC, Martín-Granizo R, Santamaría J, Infante $\mathrm{P}$, et al. Diagnóstico, prevención y tratamiento de la osteonecrosis de los maxilares por bifosfonatos. Recomendaciones de la Sociedad Española de Cirugía Oral y Maxilofacial (SECOM) 2009.

22. Mavrokokki T, Cheng A, Stein B, Goss A. Nature and frequency of bisphosphonate-associated osteonecrosis of the jaws in Australia. J Oral Maxillofac Surg. 2007;65(3):415-23. PMID: 17307586 DOI: http://dx.doi.org/10.1016/j.joms.2006.10.061 